Bull. Mater. Sci., Vol. 4, Number 3, May 1982. pp. 229-245. (C) Printed in India.

\title{
Crystal growth from solutions under microgravity environment
}

\author{
H L BHAT \\ Department of Physics, Indian Institute of Science, Bangalore 560012 , India \\ MS received 17 September 1981
}

\begin{abstract}
The growth of large and perfect single crystals are now of considerable interest because of their increasing scientific and industrial importance. Any imperfection in the crystal causcs malfunctioning, rapid aging, low reliability and low yield in manufacture. Due to the availability of earth orbiting spaceships, there is a possibility of growing these crystals with high purity. In this review paper, the author discusses mainly the growth of crystals under microgravity conditions (in spaceships) and their basic principles and also their rolative merits.
\end{abstract}

Keywords. Microgravity; convection ; gel technique ; solution growth ; flux growth ; sodimentation ; charge-transfer complexes.

\section{Introduction}

The existence of large, clear and apparently perfect crystals of many minerals in nature had attracted the attention of earlier workers in crystallography and provided an urge to emulate them in laboratory with commoner substances at their disposals. Since then, crystal growth has been the subject of considerable interest to a large number of scientific investigators. In recent years growing of large as well as perfect crystals has been further stimulated by their ever increasing demand in industry and research. Consequently, a very large number of materials have already been grown as single crystals, some with relative ease and others only after long pains-taking research. Nevertheless, there are still many substances which have defied the whale array of modern techniques on earth and which accordingly have never been seen in the single crystal form. Others, though grown by conventional techniques have never been obtained in the required size or degree of perfection. Since imperfection causes malfunctioning of devices, rapid aging, low reliability and low yield in manufacture it has always been the endeavour of material scientists to prepare purer and more perfect crystals. Crystal growth on board earth arbiting space ships may prove invaluable in achieving this objective. It is likely that this exercise may have dramatic impact on future technolagy by providing us with both new scientific knowledge and materialsnew or already known of high quality.

\section{Effect of microgravity on crystal growth}

What is it that makes crystal growth experiments under space conditions different from the ones conducted on earth? All of us know that three environmental 
parameters control growth on earth viz., (i) temperature, (ii) pressure and (iii) gravity. All desired values of temperature and pressure can be realized on earth. The main difference between pracessing on earth and in space is the absence of gravity. This can be simulated on earth only for a short period and crystal growth processes in general are of large time scale. Other factors of interest are the reduced ambient atmosphere (apprax. $10^{11}$ moleculcs $/ \mathrm{cm}^{3}$ of oxygen and hydrogen at the typical arbit reight of $200 \mathrm{~km}$ ) and the ultra high vacuum which may be obtained using the so-called molecular shield facility (Melfi 1977; Melfi et al 1976). Numerous analysis on the cansequences expected from these canditions on crystal growth have been given, most of which have been concentrated on weightlessness (see for example Proc 2nd European Symposium on Materials Science in Space, 1976). The results complemented by early experience of Skylab and ASTP flights are summarized in table 1 (Belouet 1979). We will not however go into the details of these except mentioning one or two points.

First of all the enviranment of weightlessness should minimize the gravity driven convection in fluids which causes irregular growth to occur in numerous systems and is at the origin of crystal imperfections. Convection ariscs due to the effect of gravity on density differences which are either praduced by temperature gradiants $\partial T / \partial x$ or concentration gradients $\partial C / \partial x$. In the absence of convection the transport mechanism is mainly due to diffusion or surface tension. Further, the reduced ambient atmosphere should give rise to reduced contamination and hence purer end products.

The crystal growth experiments performed during the recent ASTP mission involved growth from solution, growth by vapaur transpart as well as growth from melt. While the latter two techniques have been performed during the earlier Skylab mission the growth from solution conducted under the project title 'Crystal Growth Experiment-MAO28" was the first attempt in space to demonstrate the possibility of growing commercially important crystals by diffusion of appropriate reactants tawards each other through a region of pure water at ambient temperature (Lind 1977). We will limit ourselves to reviewing solution growth experiments only, in this article.

\section{ASTP solation growth experiment}

\subsection{Basic principle}

Since the method of growth used in the space experiment closely resembles the gel technique of crystal growth, it is appropriate to give a brief account of this technique first. Procedures for growing crystals in gels have been known since the end of last century but the metrod had fallen virtually into ablivion until a few years ago the interest in it was stimulated afresh by Henisch and his co-workers (Henisch 1970). In this method since the two or mare soluble reactants are allowed to diffuse through the gel medium and react to form an insoluble product according to the chemical reaction of the following type :

$$
\begin{aligned}
& \mathrm{A} \text { (soluble) }+\mathbf{B} \text { (soluble) }+\cdots \rightleftharpoons \mathrm{C} \text { (very slightly soluble) } \\
& \quad+\mathrm{D} \text { (soluble) }+\cdots
\end{aligned}
$$




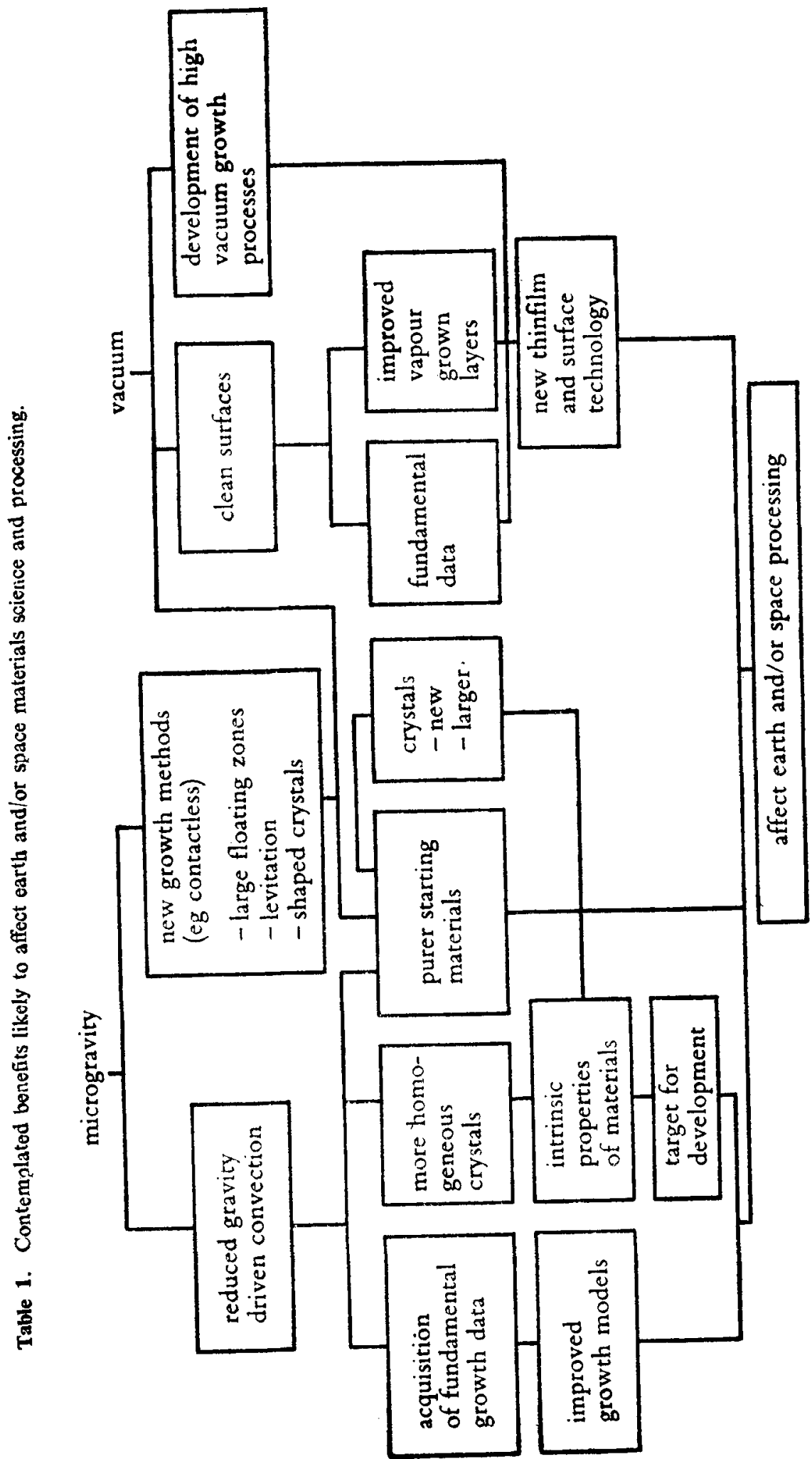


it is particularly suitable for growing crystals which have very low solubility in the solvent (usually water) that is used. This is of course growth from solution but differs from the conventional procedure by the absence of macroscopic convection currents. The gel medium prevents turbulence and remaining chemically inert provides a three-dimensional structure which permits the reagents to diffuse at a desirable controlled rate. Further, its softness and uniform nature of constraining forces that it exerts upon the growing crystals encourages orderly growth. The crystal actually grows in a cell of solution, is exposed to the solution on all sides and has no contact with the container surface except those of the flexible gel structure.

The typical experimental arrangements involved in the gel methods are exceedingly simple and are shown in figure 1. The procedure adopted for simple demonstration experiment consists of preparing a hydrogel adjusted to a specific gravity 1.05 or less which consists of ane of the reactants. Normally silica hydrogels are employed in these experiments which are prepared by mixing sodium meta silicate solution with appropriate acid. The gel may be allowed to set in a simple testtube. Once the gel is set the ather reactant may be placed over the gel without damaging its surface. Alternately a $U$ tube may be used with the gel set at the bottom and the reactants added to the arms of the $U$ tube. If the reagent incorporated in the gel is tartric acid and the second reagent is $1 \mathrm{M}$ solution of $\mathrm{CaCl}_{2}$ then in due course crystals of calcium tartrate tetrahydrate form in the gel. The first crystal can be seen near the gel solution interface within an hour or so. Good crystals however, appear further down the gel within about a week, they being of $5 \mathrm{~mm}$ average size. While the use of a test-tube limits the range of reagents and concentrations which may be added to the gel, the $U$ tube system has the disadvantages of non-linear diffusion path. Both these disadvantages can however be overcome by using a system of the type shown in figure 1c. The gel method of crystal growth like most aqueous solution growth methods are advantageous

(a)

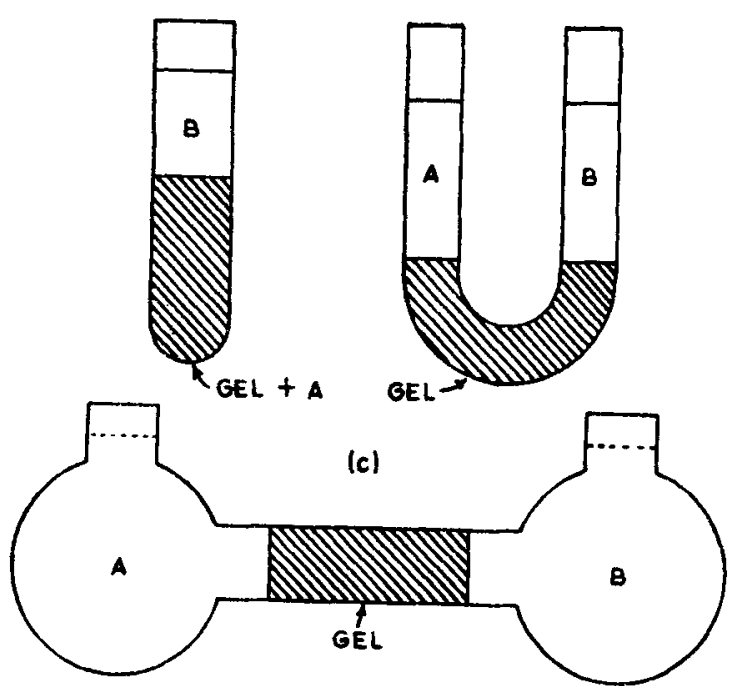

Figure 1. Typical experimental arrangements for crystal growth from gel. 
because the growth occurs at ambient (room or space craft) temperature. This minimizes the problems of thermally or mechanically induced strains, phase transformations, volatility of components, contamination by the container etc. often inherent in high temperature growth methods.

In the absence of macroscopic convection in this technique it can be reasonably assumed that the only mechanism available for the supply of solute to the growing crystal is by diffusion. That this is really the case has been amply proved by applying the Frank's volume diffusion theory which ignores the otherwise unavoidable convection currents (Frank 1950). The solution of the diffusion equation corresponds to the new phase growing at a decreasing linear rate such that the radius of the crystal is proportional to the square root of time and maintaining constant equilibrium condition at its surface. Thus one would expect the kinetics of gel grown crystals to follow a parabolic law and consequently a linear relationship between the square of the crystal size and the time. This has been experimentally verified in a number of cases. Indeed it is remarkable that the parabolic relation holds good not only for three-dimensional bulky crystals but also needle like and dendritic crystals which may be regarded as one-dimensional crystals (Liaw and Faust 1972 ; Bhat 1973).

But it is not that the gel growth technique is without any limitations. In fact it has a number of other disadvantages like excessive nucleation, gel contamination and gel instability. The method used for the experiment in space was expected to overcome all the disadvantages mentioned above while retaining the advantages of gel technique. The hypothesis for this experiment was based on the assumption that in an environment of sufficiently low gravity, convection and sedimentation become negligible. Therefore gel is not necded for suppressing these processes and can be replaced by a region of pure solvent. Diffusion becomes the predominant mechanism even with no gel present. This assumption is reasonable because typical gels are $95-96 \%$ solvent and only $4-5 \%$ solid. The advantage of allowing diffusion to be the predominant mechanism for material transport whether in gel process on earth or in microgravity process in space, is that the rate of transport can be contralled by varying the concentration of the diffusing substances and length of the diffusion path. This is important because the rate of material transport must be compatible with the rate of crystal growth which may also depend on other kinetic or heat transfer factors. A diffusion controlled process can result in better crystal quality, better prediction and control of the growth parameters involved. We will deal with these aspects in a little more detail la.ter.

\subsection{Equipment and experimental procedure}

The crystals chosen for growth experiments on ASTP flight are calcium-tartrate, calcium carbonate (calcite) and lead sulphide involving the follawing chemical reactions.

(a) Calcium tartrate-

$$
\mathrm{CaCl}_{2}+\mathrm{N} 2 . \mathrm{HC}_{4} \mathrm{H}_{4} \mathrm{O}_{6}+4 \mathrm{H}_{2} \mathrm{O} \rightleftharpoons \mathrm{Ca}_{4} \mathrm{C}_{4} \mathrm{H}_{4} \mathrm{O}_{6} \cdot 4 \mathrm{H}_{2} \mathrm{O} \downarrow+\mathrm{NaCl}+\mathrm{HCl}
$$

(b) Calcium carbonate-

$$
\mathrm{CaCl}_{2}+\left(\mathrm{NH}_{4}\right)_{2} \mathrm{CO}_{3} \rightleftharpoons \mathrm{CaCO}_{3} \downarrow+2 \mathrm{NH}_{4} \mathrm{Cl}
$$


(c) Lead sulphide-

$$
\mathrm{PbCl}_{2}+\mathrm{CH}_{3} \mathrm{CSNH}_{2}+\mathrm{H}_{2} \mathrm{O} \rightleftharpoons \mathrm{PbS} \downarrow+\mathrm{CH}_{3} \mathrm{CONH}_{2}+2 \mathrm{HCl}
$$

(Thioacetamide)

(Acetamide)

The experimental apparatus (figure 2) consisted of six specially designed and fabricated reactors. Figure 3 shows the schematic diagram of a typical reactor. Each reactor had three compartments that were separated by valves operated by the unobs at each end. Each compartment had a separate filling port. The compartments varied in length in order to facilitate different diffusion distances. The reactors were constructed of Lexan polycarbonate resin with silicone rubber gaskets and seals, parous high density polyethylene diaphragms between the compartments and aluminium knobs. The reactor covers were flat and transparent to permit photographing the progress of diffusion and crystal growth. Before the delivery of the hardware to the launch facility the reactors with their intercompartment valves in closed positions were filled with various solutions-the outer compartments with the reactant solutions and the central compartments with water. The various experimental parameters like reactant concentrations, reactor dimensions, $\mathrm{pH}$ values are shown in table 2 . These were selected on the basis of ground experiments yielding the best results.

After the joint phase of flight at approximately $109 \mathrm{hr}$ ground elapsed time, a crew man activated th: experiments by opening the intercompartment valves. Thereafter coloured photographs of the six reactors were taken at an interval of approximately $12 \mathrm{hr}$ for the remainder of the flight. Upan receipt of the flight hardware, before opening them the reactors and their contents were examined and their photographs were taken. The representative crystals were then extracted and were identified with the help of single crystal x-ray diffraction. The quality of the crystals was assessed with Lang topographic technique.

\subsection{Results}

The crystals of about expected size, quality and number formed in all the six reactors. The best crystals obtained were of calcium tartrate. This is expected because these are among the easiest to graw by the gel method. Both calcium tartrate reactors produced numerous prismatic crystals with maximum dimensions $2 \mathrm{~mm}$ and plate like crystals as long as $5 \mathrm{~mm}$. These crystals had good face developments and appeared flawless. Figure 4 shows the experimental result for calcium tartrate which are the photographs, taken after the return of the reactors to the laboratory. The quality of the crystals as confirmed by diffraction topography compared well with that of the terrestrial grown crystals. There were also a few platelets as long as $10 \mathrm{~mm}$ but of poorer quality.

Each of the two calcium carbonate reactors also contained numerous well defined clear rhombohedral crystals of $0.5 \mathrm{~mm}$ edge dimension. The crystals were very similar in appearance to earth grown calcium carbonate crystals both synthetic and natural. Although small in size they were large enough to exhibit birefringence. 


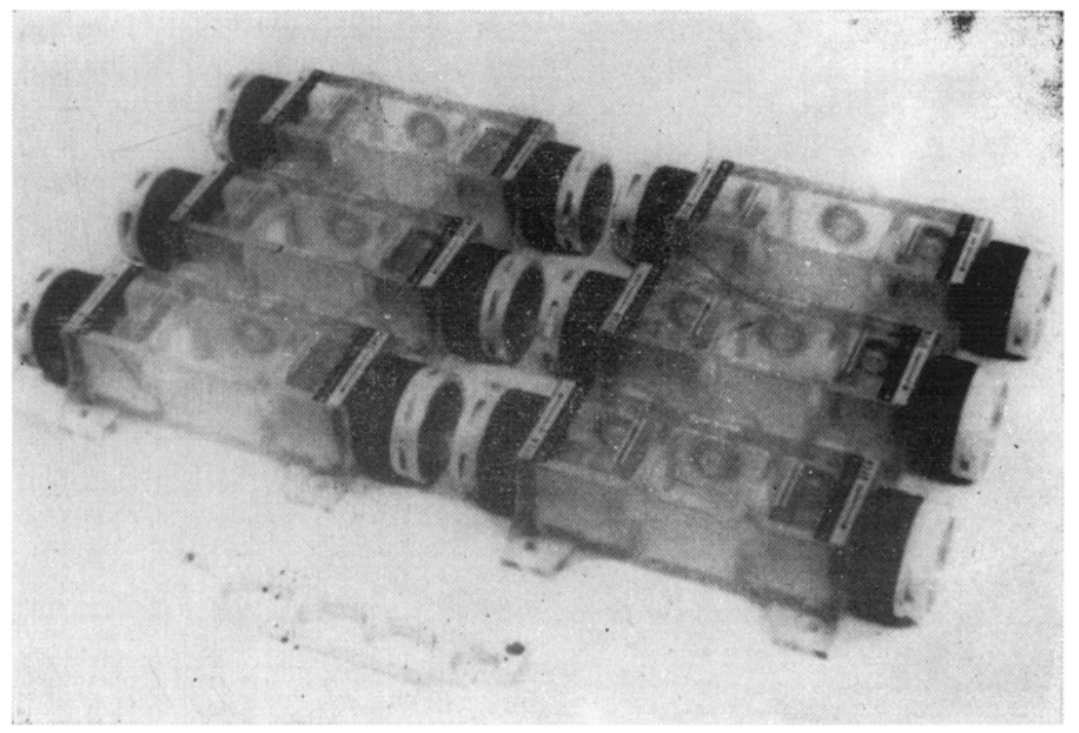

Figure 2. Flight hardware. 



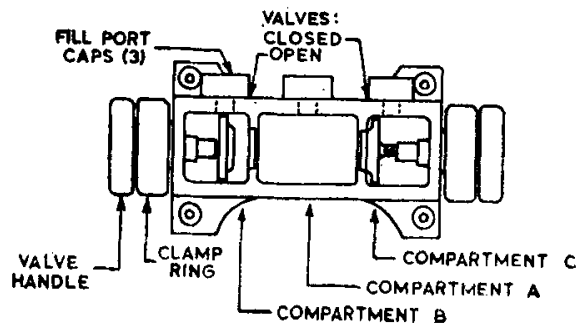

Figure 3. Schematic diagram of a reactor.

In both these cases there was no finely divided precipitate in the reactors which indicated that the mixing of the reactants and crystal growth were complete by the time of space craft re-entry, which is of course the desired result. Repeated experiments in normal gravity have shown that rapid mixing always resulted in finely divided particles which eventually settle-down at the bottom of the reactors.

The two lead sulphide experiments were less successful and yielded crystals of only $0.1 \mathrm{~mm}$ linear dimension (figure 5 ). In this case mixing and reaction were not complete at the time of re-entry. A fine precipitate was observed which continued to increase even after the return of the reactor to the laboratory. This result indicates that longer time scale is needed for the successful growth of larger $\mathrm{PbS}$ crystals under the conditions used.

The photographs of these reactors taken on board showed crystals of $\mathrm{CaCO}_{3}$ very clearly and a small number of calcium tartrate but less clearly. This indicates that most of the calcium tartrate crystals must have grown after the last photograph was taken. The flight continued for more than $6 \mathrm{hr}$ after the last series of photographs were taken and therefore it is quite possible that substantial growth occurred during this period. However, it is very likely that the growth was complete before re-entry because virtually all calcium tartrate crystals were free of reactor walls as if they were grown under low gravity condition.

The photographs taken in space also provided a record of the movement of air bubbles in reactors during flight which migrated from the expansion spaces in the filling port caps of the reactors. It is interesting to observe that the air bubbles in two calcium tartrate reactors remained stationary throughout. Bubbles in calcium carbonate and lead sulphide reactors were more mobile even though they remained stationary as long as $12 \mathrm{hr}$ interval between the two successive photographs. This difference in the mobility of the bubbles in the various reactors is not known yet. Nevertheless, the movement of the bubbles show that the accelerations experienced by the reactors during the flight were very small.

One may recall that the nucleation was excessive in all the six reactors emplayed for growing the crystals. During the flight the cabin temperature varied between approximately $297^{\circ} \mathrm{K}$ and $289^{\circ} \mathrm{K}$ which was certainly undesirable for the growth. Solubilities of the reactants and the crystalline products are strongly temperature dependent and hence this temperature variation must have given rise to excessive nucleation. Precise temperature control should definitely improve the situation. One may also initiate growth over the seeds that may be suspended in the compartment A prior to launch. Further, in choosing the various experimental parameters 


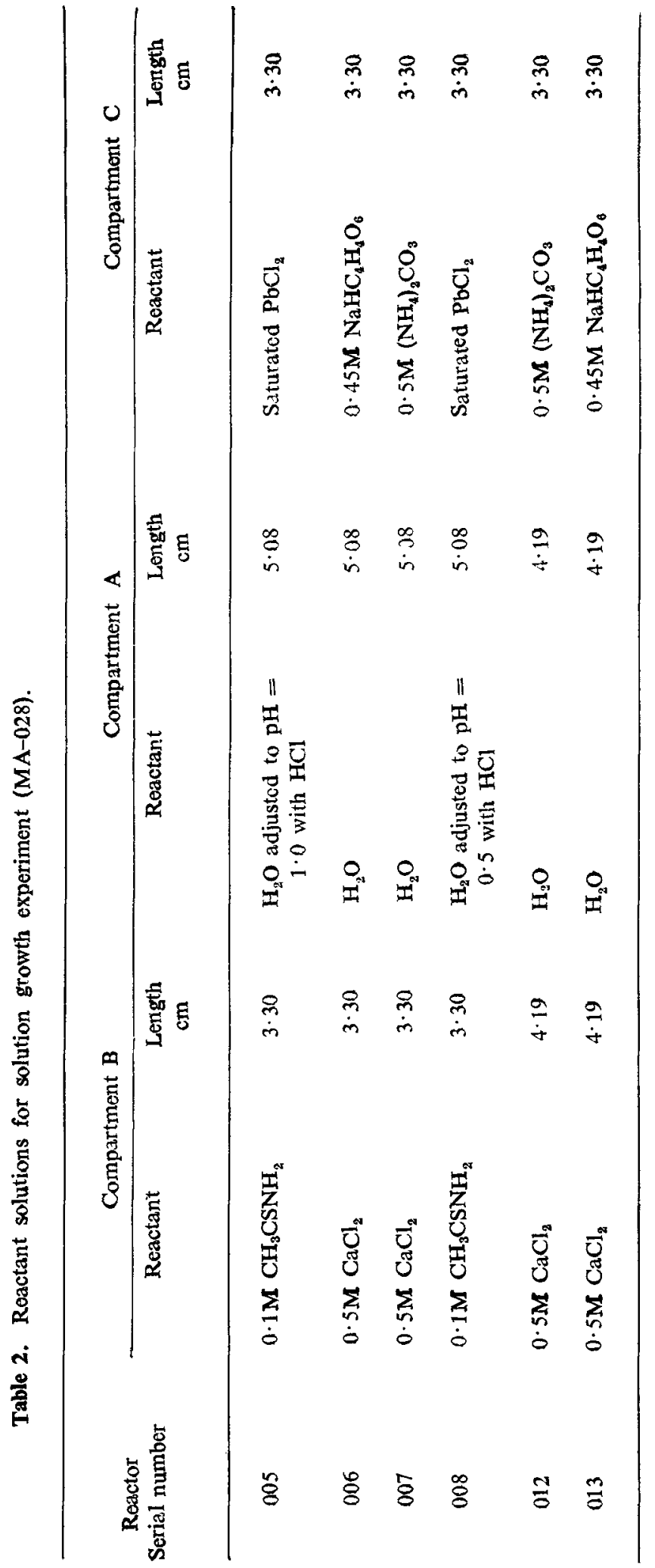



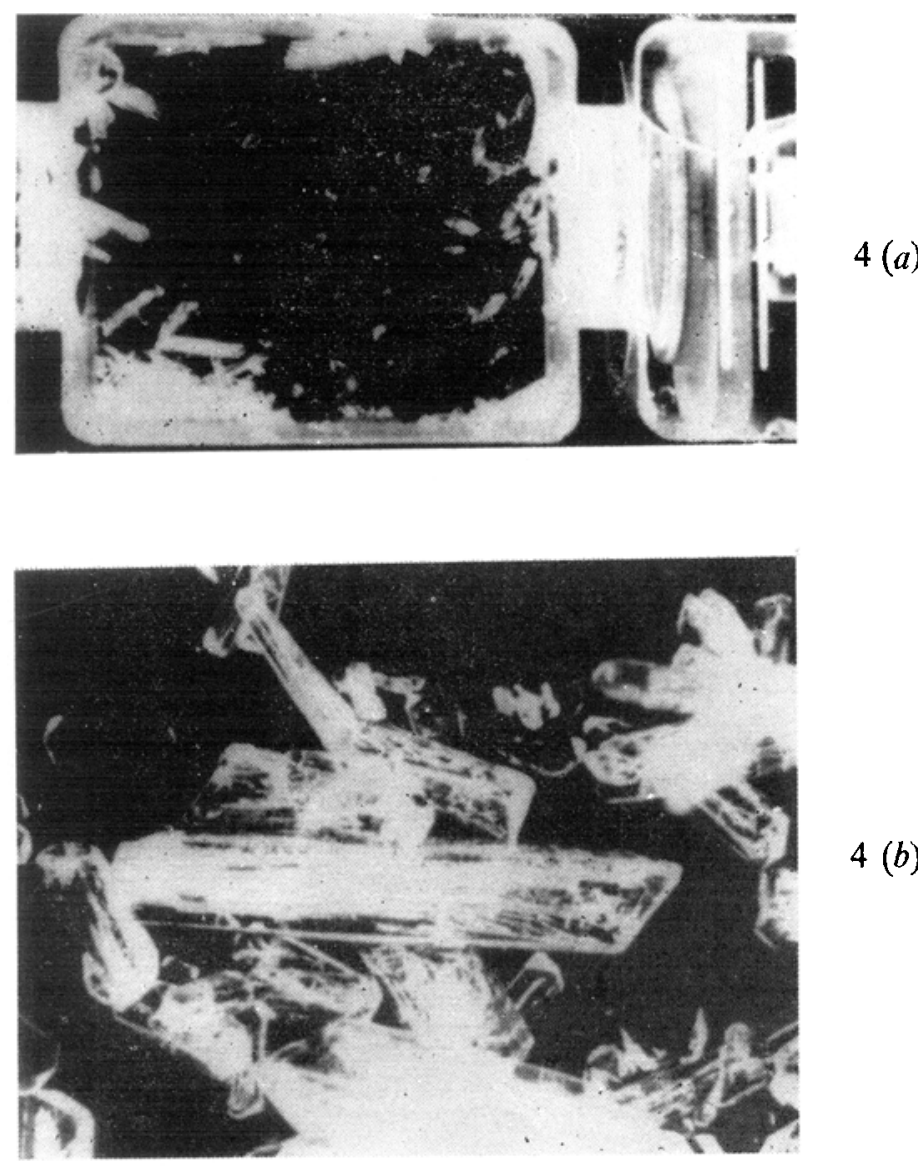

$4(b)$

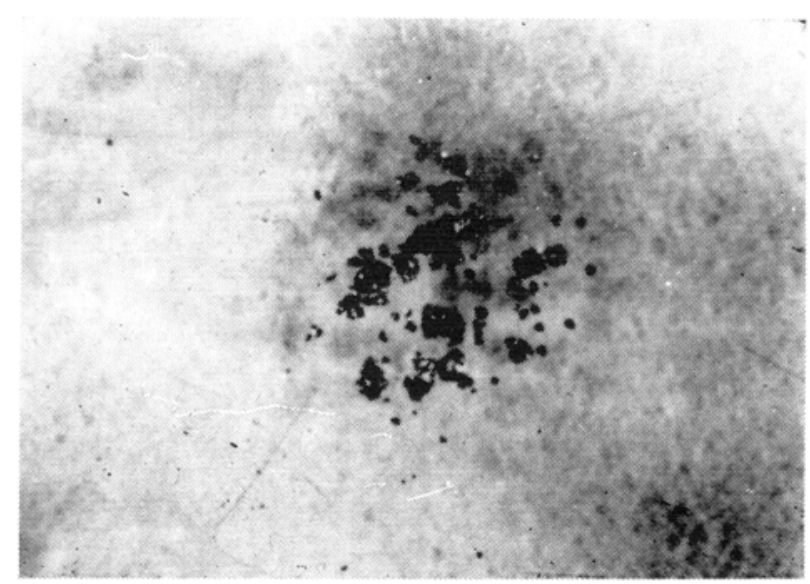

5

Figure 4. Calcium tartrate crystals grown in space (a) crystals in the reactor, (b) typical crystals.

Figure 5. Lead sulphide crystals grown in space. 

listed in table 2 , the investigator was guided more by the ground based experimental results rather than the precise knowledge of diffusion coefficients, solubilities etc. of the various materials. It is very much desirable to have a prior knowledge of these for an effective crystal growth programme in space.

\section{Growth of charge-transfer complexes in space}

Neilsen (1976) has given a simple analysis which enables one to know the time needed for the initiation of growth in terms of reactant concentration, diffusion coefficients and diffusion distance. The analysis was carried out with a view to growing charge-transfer complexes in future space programmes but may very well be adopted for the solution growth of the type described earlier.

A characteristic feature of the charge-transfer compounds is that their properties seem to depend very much on the way one grows them in the sense that only crystals with the highest perfection show the desired electronic properties of onedimensional conductivity. The best known example is TTF-TCNQ (tetrathiofulvelene-tetracyanoquino-dimethane). The data for six different crystals of these showed that the narmalized conductivity at $T_{c}$ varied between 15 and 150 (Shehegolev 1972 ; Cohen et al 1974). The crystals of TTF-TCNQ are normally grawn from a solution of acetonitrile containing TTF and TCNQ in required quantities. Here TTF acts as an electron donor (D) and TCNQ as electron acceptor (A). The DA complex is formed by transfer of an electronic charge from $D$ to $A$ and hence the name. Anywhere in the solution where the product of $D$ and A concentration exceeds the DA solubility product which is low compared to $D$ and $A$ solubilities the DA complex will precipitate and hence it is very important to the crysta.l grower that the solubility praduct exceeded only near the growing crystal and not anywhere else. This is nearly impossible to achieve on earth because of density induced convection currents. Actual gel growth of these complexes have been tried extensively in the laboratory but always with the result that the crystals at best would grow at the top of the gel. One believes that the weightless condition in space pravides a unique opportunity to achieve the required perfection in reasonably large crystals.

Under microgravity condition we may consider an experimental arrangement similar to the one used in solution growth with a seed crystal DA at the central compartment and the two reactants on either side of the seed crystal as shown in figure 6. These reactants are in equilibrium with their respective solutes such that they are saturated. Referring only to the left side of the ampule let $\boldsymbol{C}_{\boldsymbol{i}}$ and $\boldsymbol{C}_{\text {. }}$ be the initial and final $D$ concentrations respectively and DA crystal and $\boldsymbol{C}_{0}$ the saturated $D$ concentration at D. One can directly apply the Fick's laws

$$
I=-D \frac{d C}{d x} \text { and } \frac{d C}{d t}=D \frac{d^{2} C}{d X^{2}}
$$

(here $D$ is the diffusion coefficient),

to the above system to obtain the following solution

$$
\frac{C_{0}-C_{i}}{C_{0}-C_{i}}=\frac{\triangle C_{b}}{\triangle C_{0}}=\operatorname{erfc} x / 2 \sqrt{D t}
$$




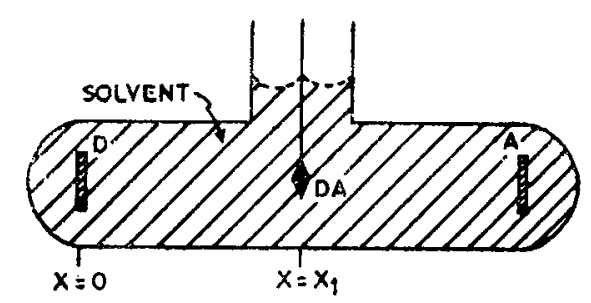

Figure 6. Schematic diagram of the arrangement to grow charge transfer crystals

The approximation made here is that the diffusion takes place with constant surface concentration $C_{0}$ into a volume with initial homogeneous concentration $C_{i}$ in one dimension only. Further one assumes that the dissolution and grawth are diffusion controlled and that no volume or temperature change accurs during these pracesses.

At time $t=0$ both D and DA crystal start dissolving and a current of D malecules (alsa A molecules) will leave DA crystal. As time advances a situation will be reached at $t=t_{c}$, when there will be no net current at DA crystal and this is represented by the curve 2 , in figure 7 . The $\mathrm{D}$ distribution at $t<t_{c}$ will be as shown by the curve 1 in the same figure. The time $t_{0}$ represents the turning point of the current and thus the time necessary for the initiation of growth. Thereafter there will be a resultant flow of D molecules from D to DA crystal as is represented by the curve 3. An expression for $t_{c}$ may be obtained by superimpasing the two independent solutions

$$
\Delta C_{0}(x)=\Delta C_{0} \operatorname{erfc} \frac{x}{2 \sqrt{D t}}
$$

and

$$
\Delta C_{1}(x)=\Delta C_{1} \operatorname{erfc} \frac{x_{1}-x}{2 \sqrt{D t}}
$$

Setting the diffusion current $I=0$ at $x=x_{1}$ one obtains the simplest relation between $x_{1}$ and $t_{0}$ as

$$
x_{1}^{2}=4 D t_{0} \ln \frac{C_{0}-C_{i}}{C_{1}-C_{i}},
$$

where $C_{1}$ may be chosen as the square root af the solubility product. Obviously this does not give the complete picture since only the product has to be exceeded in order to obtain precipitation. Nevertheless, for a fixed temperature and a given apparatus this analysis will tell the crystal grower appraximately at what time he should expect growth to initiate. One can now make use of this to optimize the experimental conditions for the effective utilization of the limited time available in the space programme. A series of experiments using this isotbermal reaction technique by Nielsen are expected to be flown on the first Spacelab mission to explore the possibilities of growing charge-transfer crystals as well as materials of biological importance under microgravity condition. A preliminary design of his experimental set up is shown in figure 8. 


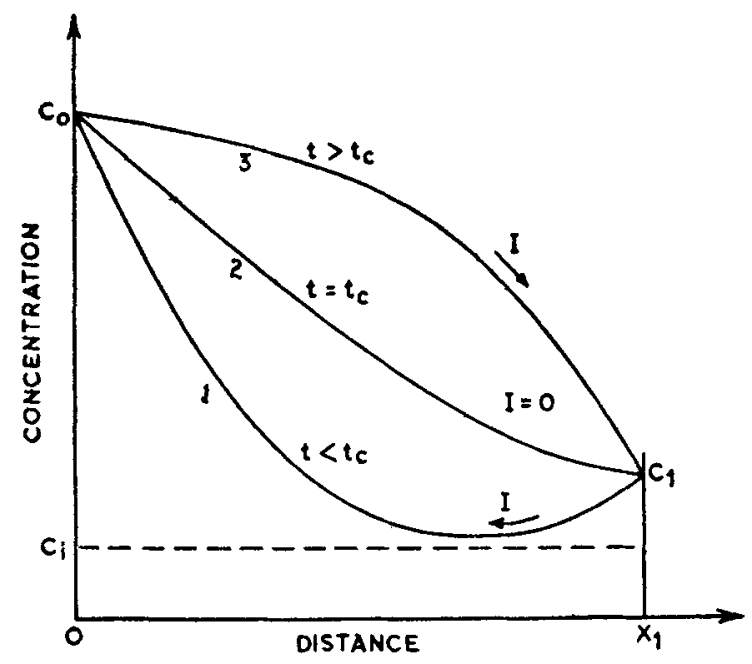

Figure 7. Concentration diagram for $D$ molecules (or left hand side of the ampoule only).

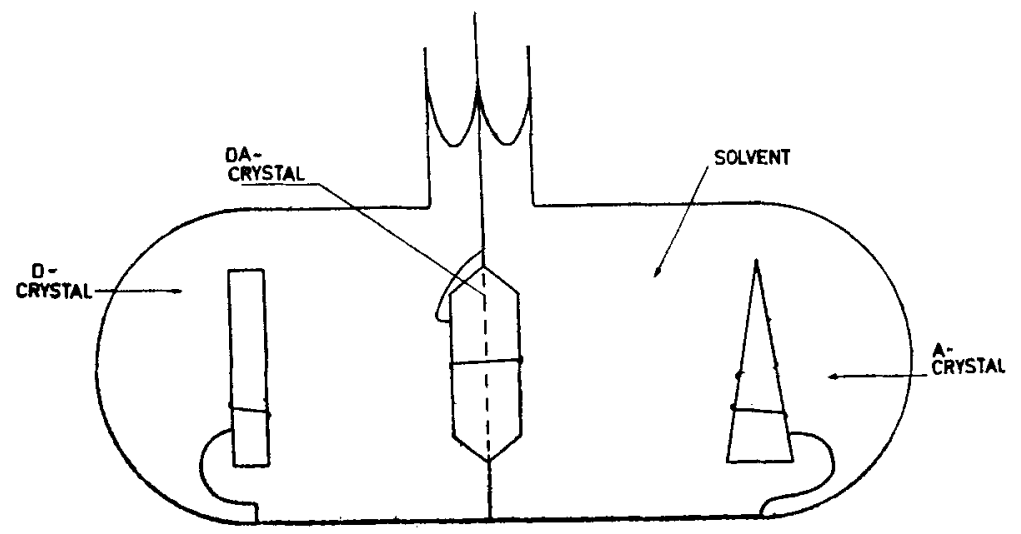

Figure 8. Preliminary design of the ampoule for space lab.

\section{High temperature solution growth}

Finally a few remarks about crystal growth from high temperature salution. In recent years, the requirement of materials like YIG and GaAs for device applicatians has resulted in a cansiderable improvement of high temperature salution techniques for reprodusible results. Nevertheless, the crystals af desired purity and perfection are very hard to achieve. It has been the experience of the crystal grower that in flux growth involving spontaneous nucleation, excessive nucleation resulting in a large number of small crystals is a big problem. Further, on account of density differences between the crystal and the flux material, sedimentation always occurs. Growth of crystals from high temperature solution in space should in principle improve the situation considerably because the problems associated with the convection and sedimentation are absent there. But whether it is economically a feasible propasition is a matter to be considered. On the other hand one can definitely make use of the opportunity to. determine certain 
properties of high temperature solution. Accurate determination of these parameters is important if the growth apparatus to be designed and used shauld be on scientific principles rather than guess work.

In recent years a number of theoretical analysis of growth rate controlling mechanisms, and conditions for stable growth have been carried out in terms of various solution parameters. The expressions for the growth rates also contain certain other parameters of technical importance. Elwell (1976) has loaked into these parameters from the point of view of accurate measurements and the passible advantages of measuring them under microgravity. He comes to the conclusion that three parameters, viz., solute diffusivity, thermal diffusivity and crystal surface energy are particularly warthy of investigations in mini-gravity of which the former appears to be the most impartant. The difficulty in measuring the diffusion coefficient on earth is illustrated by the spread in the $D$ value of garnet solutes in $\mathrm{PbO} / \mathrm{B}_{2} \mathrm{O}_{3}$ solvent. The literature values vary from $(5 \pm 1) 10^{-7} \mathrm{~cm}^{2} / \mathrm{sec}$. (Knight et al 1974) to $1.1-2.4 \times 10^{-5} \mathrm{~cm}^{2} / \mathrm{sec}$. (Morgan 1974).

A particularly simple method of measuring the diffusion caefficient in microgravity for magnetic garnet salutes is due to $\mathrm{J} \mathrm{C}$ Brice and $\mathrm{T} \mathbf{H}$ Bruton (MS-5) (Nielsen 1976) and the proposed diffusion cell is as shown in figure 9. The method consists in holding a garnet crystal in contact with the sample salvent at constant temperature for a few hours and then cool the cartridge containing the sample rapidly. The distribution of the solute in the salidified sample may then be determined using radio active tracer or electron microprobe analysis. A major source of error in the experiment is the segregation of solute as the solution cools and it is important that this phenomenon is thoroughly studied in the laboratory priar to the flight.

There is an apprehension regarding the usefulness of solution growth progtamme in space which arises mainly from the mass transport consideration. In the absence of thermal convection, diffusion and surface tension will be dominant. Material transport by diffusion is smaller by one or two orders of magnitude than that by convection. This results in a very low growth rate for solution growth and hence makes the technique unattractive. At the same time, the isothermal solution reaction technique described in $\$ \S$ and 4 has the merit of requiring no electrical power apart from a few watts for temperature control and little operator intervention. Accordingly, for routine crystal growth experiments in space this method appcars to be a strang cantender.

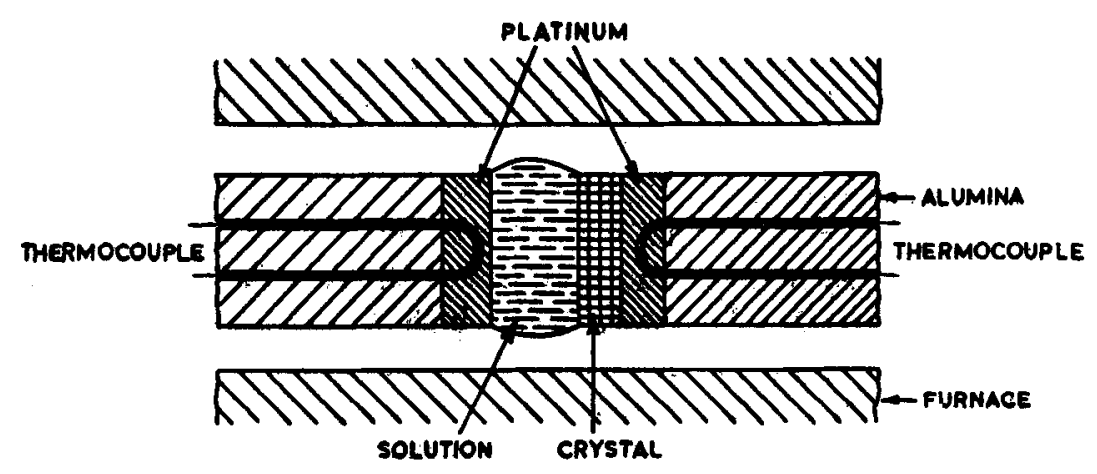

Figure 9. Cell for diffusion coefficient measurement. 


\section{Conclusion}

Crystal growth experiments conducted in space so far have not given exciting results. However it should be noted that many previous experiments in space have been hastily devised or performed under severe restriction. While the individual experiments are subject to criticism, this exercise has resulted in a number of interesting side results which will be very useful for earth bound experiments. For example, the develapment of a mirror furnace will result in the improved furnace design with great saving of energy. An estimate says that if everyone on earth used the types of furnaces designed and developed for space a substantial cast of the space programme would be recavered in savings on electricity.

Obviously, manufacturing in space cannot solve all the technical and especially the economic problems. The possibility for crystal growth under micragravity offered by the space programmes are 'one-off' experiments. Hence, the experiments for the future space missions should be carefully planned so that they nat only help us in making crystal growth in space feasible but also in achieving better understanding of many problems assaciated with crystal growth on earth and perhaps to solve them. Of the two, the most valuable rale of space programme would be of course, the better understanding of the crystal growth phenomena in general which will help the transition of this subject from an art which even now depends very much on individual skill and instinct to a science.

\section{Acknowledgement}

The author thanks Prof.P S Narayanan for his keen interest and kind encouragement in the preparation of this article.

\section{References}

Belouet C 1979 Thin Solid Films 581

Bhat H L 1973 Studies on growth and defect properties of Barite group crystals, Ph.D. Thesis, Sardar Patel University, Vallabh Vidyanagar

Cohen M J, Coleman L B, Carito A F and Heeger A J 1974 Phys. Rev. B10 1298

Elwell D 1976 Proc. 2nd European symposium on materials sciences in Space, Frascati, Italy 6-8 April, p. 249

Frank F C 1950 Proc. R. Soc. A201 586

Henisch H K 1970 Crystal growth in gels, ed. (The Pennsylvania State University Press, University Park and London)

Knight S, Hewitt B S, Rode D L and Blank S L 1974 Mater. Res. Bull. 9895

Liaw H M and Faust J W Jr 1972 J. Crystal Growth 13/14 471

Lind M D 1977 Apolo-Soyuz test project summary science report NASA, SP412, 1555

Melfi L T Jr 1977 Acta Astronaut 4801

Meli L T Jr, Outlaw R A, Hueser J E and Brock F J 1976 J. Vac. Sci. Technol. 13698

Morgan A E 1974 J. Crystal Growth 27266

Nielsen K F 1976 Proc. 2nd European symposium on materials sciences in space, Frascati, Italy 6-8 April, p. 255

Shehegolev I F 1972 Phys. Status Solidi A12 9

Proc. 2nd European symposium on materials sciences in space, Frascati, Italy, 6-8 April 1976 